\title{
ON NONLOCAL PROBLEMS FOR ORDINARY DIFFERENTIAL EQUATIONS AND ON A NONLOCAL PARABOLIC TRANSMISSION PROBLEM
}

\author{
M. DENCHE \\ Université de Constantine \\ Institut de Mathematiques \\ Constantine \\ Algeria
}

(Received July, 1997; Revised August, 1998)

\begin{abstract}
In the present paper we study nonlocal problems for ordinary differential equations with a discontinuous coefficient for the high order derivative. We establish sufficient conditions, known as regularity conditions, which guarantee the coerciveness for both the space variable and the spectral parameter, as well as guarantee the completeness of the system of root functions. The results obtained are then applied to the study of a nonlocal parabolic transmission problem.

Key words: Nonlocal Problems, Ordinary Differential Equations, Parabolic Differential Equation, Boundary and Transmission Conditions, Coerciveness Estimate, Completeness, Root Functions, Elementary Solutions.
\end{abstract}

AMS subject classifications: 34A30, 34B05, 34L40, 47E05, 35K20, $35 \mathrm{~K} 99$.

\section{Introduction}

Many physical problems, the problem of heat and mass diffusion in anisotropic media, diffraction problems, and others lead to the study of equations with a discontinuous coefficient for the high order derivative [13]. In the present paper, we start with the study of boundary value problems for ordinary differential equations with a discontinuous coefficient for the high order derivative and with boundary conditions containing abstract functionals. We establish sufficient conditions of regularity type which guarantee the coerciveness for both the space variable and the spectral parameter, and which also guarantee the completeness of root functions. Regular problems for differential operators are studied in $[3,9,10]$. Completeness of the system of root functions for differential operators with functional boundary conditions is analyzed in $[4,6,11,14]$. The coefficient of the high order derivative is assumed to be constant in $[6,11]$, whereas it is assumed to be continuous in [14]. The results obtained are used to show the existence and uniqueness of the solution, of a mixed 
problem for a parabolic partial differential equation with a discontinuous coefficient for the high order derivative, and multipoint boundary values and transmission conditions containing abstract functionals. They are also used to show the completeness of elementary solutions of this given mixed problem. Thus, the study is reduced to a Cauchy problem for a parabolic abstract differential equation, where the analysis of the operator coefficient is given in detail in this paper.

\section{Preliminaries}

Let $W_{q}^{m}(a, b)$ denote a Sobolev space, defined so that

$$
W_{q}^{m}(a, b)=\left\{u \in L_{q}(a, b): D^{\alpha} u \in L_{q}(a, b) ; \alpha \leq m\right\}, q \in(1, \infty) .
$$

Lemma 1: For $u \in W_{q}^{m}(a, b)$, we have the following inequality [2]:

$$
\max _{x \in[a, b]}\left|u^{(k)}(x)\right| \leq c\left(h^{1-\chi}\left\|u^{(m)}\right\|_{L_{q}(a, b)}+h^{-\chi}\|u\|_{L_{q}(a, b)}\right)
$$

where $0 \leq k<m ; 0<h<h_{0} ; \chi=\frac{1}{m}\left(k+\frac{1}{q}\right) ; q \in(1, \infty)$.

Lemma 2: For $y \in W_{2}^{k}(a, b)$ and $\lambda \in \mathbb{C}$, we have

$$
|\lambda|^{k-s}\|y\|_{W_{2}^{s}(a, b)} \leq C_{k s}\left(\|y\|_{W_{2}^{k}(a, b)}+|\lambda|^{k}\|y\|_{L_{2}(a, b)}\right), \quad 0 \leq s \leq k .
$$

Remark 1: Lemma 2 is a particular case of a result given in [1].

\section{Nonlocal Problem for an Ordinary Differential Equation}

In $[0,1] \backslash b$, we consider the equation

$$
L(\lambda) u=-a(x) u^{\prime \prime}(x)+(B u)(x)-\lambda u(x)=f(x)
$$

where $\lambda$ is a complex parameter. To equation (1) we add the boundary conditions:

$$
\left\{\begin{array}{l}
L_{1} u=\alpha_{11} u^{\left(K_{1}\right)}(0)+\beta_{11} u^{\left(K_{1}\right)}(1)+\sum_{p=1}^{N} \delta_{1 p} u^{\left(K_{1}\right)}\left(x_{1 p}\right)+T_{1} u=f_{1} \\
L_{2} u=\alpha_{21} u^{\left(K_{2}\right)}(0)+\beta_{21} u^{\left(K_{2}\right)}(1)+\sum_{p=1}^{M} \delta_{2 p} u^{\left(K_{2}\right)}\left(x_{2 p}\right)+T_{2} u=f_{2}
\end{array}\right.
$$

and we include the transmission conditions:

$$
\left\{\begin{array}{c}
L_{3} u=\alpha_{31} u(b-0)+\beta_{31} u(b+0)+T_{3} u=f_{3} \\
L_{4} u=\alpha_{41} u^{\prime}(b-0)+\beta_{41} u^{\prime}(b+0)+T_{4} u=f_{4}
\end{array}\right.
$$


where $a(x)=\left\{\begin{array}{c}a_{0} \text { for } x \in(0, b] \\ a_{1} \text { for } x \in(b, 1]\end{array}, a_{0}, a_{1} \neq 0, x_{1 p}, x_{2 p} \in(0, b) . \quad B\right.$ is a linear operator and each $T_{\nu}$ is a linear functional $(\nu=\overline{1,4}) . \quad \alpha_{j 1}, \beta_{j 1}, \delta_{i p} \in \mathbb{C} ; K_{i}=0,1 ; i=\overline{1,2} ; j=\overline{1,4}$; $p=\frac{\nu}{1, N}$. Let

$$
\bar{\omega}:=\max \left\{\arg a_{0}, \arg a_{1}\right\}, \underline{\omega}:=\min \left\{\arg a_{0}, \arg a_{1}\right\}
$$

and

$$
S_{\varepsilon}:=\{\lambda \in \mathbb{C}: \varepsilon+\bar{\omega}<\arg \lambda<2 \pi-\varepsilon+\underline{\omega}\}
$$

\subsection{Coerciveness of a Principal Boundary Value Problem}

Consider, in $[0,1] \backslash b$ the simplified problem:

$$
L_{0}(\lambda) u=-a(x) u^{\prime \prime}(x)-\lambda u(x)=f(x)
$$

with

$$
\left\{\begin{array}{l}
L_{10} u=\alpha_{11} u^{\left(K_{1}\right)}(0)+\beta_{11} u^{\left(K_{1}\right)}(1)=f_{1} \\
L_{20} u=\alpha_{21} u^{\left(K_{2}\right)}(0)+\beta_{21} u^{\left(K_{2}\right)}(1)=f_{2}
\end{array}\right.
$$

and with

$$
\left\{\begin{array}{c}
L_{30} u=\alpha_{31} u(b-0)+\beta_{31} u(b+0)=f_{3} \\
L_{40} u=\alpha_{41} u^{\prime}(b-0)+\beta_{41} u^{\prime}(b+0)=f_{4}
\end{array}\right.
$$

Theorem 1: Suppose that

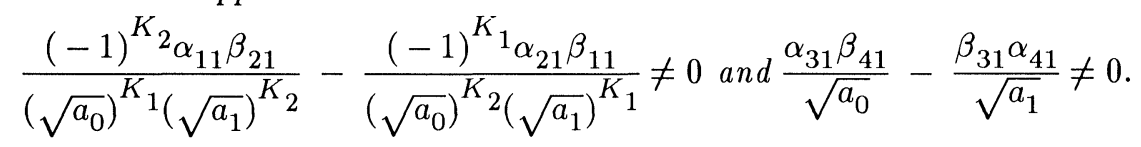

Then for each $\varepsilon>0$ there exists $R_{\varepsilon}>0$, such that for any complex number $\lambda \in S_{\varepsilon}$ with $|\lambda|>R_{\varepsilon}$, the operator

$$
\ell_{0}(\lambda): u \mapsto\left(L_{0} u, L_{10} u, L_{20} u, L_{30} u, L_{40} u\right),
$$

from $W_{q}^{l}(0, b) \times W_{q}^{l}(b, 1)$ onto $W_{q}^{l-2}(0, b) \times W_{q}^{l-2}(b, 1) \times \mathbb{C}^{4}$, is an isomorphism. Furthermore, for any such $\lambda$ and any solution of problem (4)-(6), the following estimate holds.

$$
\begin{aligned}
& \sum_{k=0}^{l}|\lambda|^{\frac{1}{2}(l-k)}\|u\|_{W_{q}^{k}(0, b) \times W_{q}^{k}(b, 1)} \\
& \leq C(\varepsilon)\left(\|f\|_{W_{q}^{l-2}(0, b) \times W_{q}^{l-2}(b, 1)}+|\lambda|^{\frac{1}{2}(l-2)}\|f\|_{L_{q}(0, b) \times L_{q}(b, 1)}\right. \\
& \left.\quad+\sum_{\nu=1}^{4}|\lambda|^{\frac{1}{2}\left(l-k_{\nu}-\frac{1}{q}\right)}\left|f_{\nu}\right|\right)
\end{aligned}
$$


Proof: Clearly, operator $\mathcal{L}_{0}(\lambda)$ acts linearly and continuously from $W_{q}^{l}(0, b) \times$ $W_{q}^{l}(b, 1)$ into $W_{q}^{l-2}(0, b) \times W_{q}^{l-2}(b, 1) \times \mathbb{C}^{4}$. Let us show that, for every $f \in$ $W_{q}^{l-2}(0, b) \times W_{q}^{l-2}(b, 1)$ and for every $f_{\nu} \in \mathbb{C}, \nu=\overline{1,4}$, problem (4)-(6) has a unique solution belonging to $W_{q}^{l}(0, b) \times W_{q}^{l}(b, 1)$.

We seek the solution $y$ of problem (4)-(6) in the form $y=y_{1}+y_{2}$, with $y_{1}=\left(u_{1}, u_{2}\right)$ and $y_{2}=\left(u_{3}, u_{4}\right)$, so that $y_{1}$ will be a restriction on $[0,1]$ of the solution $\widetilde{y}_{1}$ to the equation

$$
L_{0}(\lambda) \tilde{y}_{1}=\tilde{f}(x), \quad x \in \mathbb{R}
$$

where

$$
\tilde{f}(x)=\left\{\begin{array}{cc}
f(x), & x \in[0,1] \\
0, & x \notin[0,1]
\end{array}\right.
$$

and $y_{2}$ will be the solution of the problem:

$$
\left\{\begin{array}{c}
L_{0}(\lambda) y_{2}=0 \\
L_{\nu 0} y_{2}=-L_{\nu 0} y_{1}+f_{\nu}, \nu=\overline{1,4}
\end{array}\right.
$$

By applying a Fourier transform, denoted by $\mathscr{F}$, to equation (8) and by making the change of variable $\lambda=-a_{0} \rho^{2}$ and $\lambda=-a_{1} \rho^{2}$, we obtain

$$
\left\{\begin{array}{l}
a_{0}\left[(i \sigma)^{2}-\rho^{2}\right] \mathscr{F} u_{1}=-\mathscr{F} \tilde{f}_{1} \\
a_{1}\left[(i \sigma)^{2}-\rho^{2}\right] \mathscr{F} u_{2}=-\mathscr{F} \tilde{f}_{2}
\end{array}\right.
$$

As $\frac{\pi}{2}+\varepsilon<\arg \rho<\frac{3 \pi}{2}-\varepsilon$ and $\sigma \in \mathbb{R}$, using geometrical arguments, we have

$$
\left|(i \sigma)^{2}-\rho^{2}\right| \geq c(\varepsilon)\left(|\sigma|^{2}+|\rho|^{2}\right) .
$$

Then $u_{j}^{(k)}=\mathscr{F}^{-1} \mathcal{F}_{u_{j}}^{(k)}=\mathscr{F}^{-1}(i \sigma)^{k} \mathcal{F}_{j}=-\mathscr{F}^{-1}\left(\underline{i \sigma)^{k}} a_{j-1}^{-1}\left[(i \sigma)^{2}-\rho^{2}\right]^{-1} \mathcal{F}_{\tilde{f}}\right.$. Now, let $T_{k}^{j}(\sigma, \lambda)=\rho^{2-k^{j}}(i \sigma)^{k} a_{j-1}^{-1}\left[(i \sigma)^{2}-\rho^{2}\right]^{-1} j=\overline{1,2}$ and $k=\overline{0,2}$. Clearly, each $T_{k}^{j}(\sigma, \lambda)$ is continuously differentiable with respect to $\sigma$ in $\mathbb{R}$ and, from inequality (11), we get

$$
\left|T_{k}^{j}(\sigma, \lambda)\right| \leq c(\varepsilon),\left|\frac{\partial T_{k}^{j}(\sigma, \lambda)}{\partial \sigma}\right| \leq \frac{c(\varepsilon)}{|\sigma|} .
$$

Then, by virtue of the Mikhlin theorem [3], the function $T_{k}^{j}(\sigma, \lambda)$ is a Fourier multiplier of type $(q, q)$. Hence, if $\tilde{f} \in L_{q}(\mathbb{R}) \times L_{q}(\mathbb{R})$, then a function $\widetilde{y}_{1}$ is a solution to equation (8) belonging to $W_{q}^{2}(\mathbb{R}) \times W_{q}^{2}(\mathbb{R})$, and so we have

$$
\sum_{k=0}^{2}|\rho|^{2-k}\left\|\tilde{y}_{1}^{(k)}\right\|_{L_{q}(\mathbb{R}) \times L_{q}(\mathbb{R})} \leq c(\varepsilon)\|\tilde{f}\|_{L_{q}(\mathbb{R}) \times L_{q}(\mathbb{R})^{\cdot}}
$$

Using (12) and Lemma 2 with a recursive argument, we can easily show that if $\tilde{f} \in$ $W_{q}^{l-2}(\mathbb{R}) \times W_{q}^{l-2}(\mathbb{R})$, then $\tilde{y}_{1} \in W_{q}^{l}(\mathbb{R}) \times W_{q}^{l}(\mathbb{R})$ and the following estimate is valid 
$\sum_{k=0}^{l}|\lambda|^{\frac{1}{2}(l-k)}\left\|\tilde{y}_{1}\right\|_{W_{q}^{k}(\mathbb{R}) \times W_{q}^{k}(\mathbb{R})}$

$$
\leq c(\varepsilon)\left(\|\tilde{f}\|_{W_{q}^{l-2}(\mathbb{R}) \times W_{q}^{l-2}(\mathbb{R})}+|\lambda|^{\frac{1}{2}(l-2)}\|\tilde{f}\|_{L_{q}(\mathbb{R}) \times L_{q}(\mathbb{R})}\right)
$$

Thus, equation (8) possesses a unique solution $\tilde{y}_{1} \in W_{q}^{2}(\mathbb{R}) \times W_{q}^{2}(\mathbb{R})$ and for $y_{1}$ we get

$$
\begin{aligned}
& \sum_{k=0}^{l}|\lambda|^{\frac{1}{2}(l-k)}\left\|y_{1}\right\|_{W_{q}^{k}(0, b) \times W_{q}^{k}(b, 1)} \\
& \quad \leq c(\varepsilon)\left(\|f\|_{W_{q}^{l-2}(0, b) \times W_{q}^{l-2}(b, 1)}+|\lambda|^{\frac{1}{2}(l-2)}\|f\|_{L_{q}(0, b) \times L_{q}(b, 1)}\right) .
\end{aligned}
$$

Now, let us establish that for any complex number $f_{\nu} \in \mathbb{C}, \nu=\overline{1,4}$, problem (9)-(10) has a unique solution $y_{2}$ belonging to $W_{q}^{l}(0, b) \times W_{q}^{l}(b, 1)$; and for an estimate of the solution, we have $L_{0}(\lambda) y_{2}=0$, which is equivalent to

$$
\left\{\begin{array}{l}
-a_{0} u_{3}^{\prime \prime}-\lambda u_{3}=0 \text { in }[0, b) \\
-a_{1} u_{4}^{\prime \prime}-\lambda u_{4}=0 \text { in }(b, 1]
\end{array}\right.
$$

Setting $\rho_{0}=\sqrt{\frac{-\lambda}{a_{0}}}$ and $\rho_{1}=\sqrt{\frac{-\lambda}{a_{1}}}$, we get that the general solutions of the equations of (14) are, respectively, $u_{3}=c_{1} \exp \rho_{0} x+c_{2} \exp \left[-\rho_{0}(x-b)\right]$ and $u_{4}=c_{3} \exp \rho_{1}(x-b)$ $+c_{4} \exp \left[-\rho_{1}(x-1)\right]$. Substituting these expressions into the conditions of $(10)$, we obtain a system for $c_{k}, k=\overline{1,4}$. By a straightforward computation, it is easy to see that the determinant of this system is of the form:

$$
\Delta=\theta+R\left(\rho_{0}, \rho_{1}\right)
$$

where $\theta=\left[a_{11} \beta_{21} \rho_{0}^{k}\left(-\rho_{1}\right)^{k_{2}}-\alpha_{21} \beta_{11} \rho_{0}^{k_{2}}\left(-\rho_{1}\right)^{k_{1}}\right] \quad\left(\alpha_{31} \beta_{41} \rho_{1}+\beta_{31} \alpha_{41} \rho_{0}\right) \quad$ and $R\left(\rho_{0}, \rho_{1}\right) \rightarrow 0$ for $\left|\rho_{0}\right|,\left|\rho_{1}\right| \rightarrow \infty$, in $S_{\varepsilon}$. According to the assumption, we have $\theta \neq 0$.

Again, by straightforward computation, we find that each $c_{i}$ is of the form: $c_{i}=$ $\frac{\theta_{i}+R_{i}\left(\rho_{0}, \rho_{1}\right)}{\theta+R\left(\rho_{0}, \rho_{1}\right)}$, where $\theta_{i}$ is obtained from $\theta$ by replacing the column $i$ with the column formed by right-hand sides of the boundary conditions of $(10)$ such that $R_{i}\left(\rho_{0}, \rho_{1}\right) \rightarrow$ $0, i=\overline{1,2}$ for $\left|\rho_{0}\right|,\left|\rho_{1}\right| \rightarrow \infty$, in $S_{\varepsilon}$. Substituting these values in the expressions of $u_{3}$ and $u_{4}$, we find that problem (9)-(10) has a unique solution, given by

$$
\begin{gathered}
u_{3}=\frac{\theta_{1}+R_{1}\left(\rho_{0}, \rho_{1}\right)}{\theta+R\left(\rho_{0}, \rho_{1}\right)} \exp \rho_{0} x+\frac{\theta_{2}+R_{2}\left(\rho_{0}, \rho_{1}\right)}{\theta+R\left(\rho_{0}, \rho_{1}\right)} \exp \left[-\rho_{0}(x-b)\right] \\
u_{4}=\frac{\theta_{3}+R_{3}\left(\rho_{0}, \rho_{1}\right)}{\theta+R\left(\rho_{0}, \rho_{1}\right)} \exp \rho_{1}(x-b)+\frac{\theta_{4}+R_{4}\left(\rho_{0}, \rho_{1}\right)}{\theta+R\left(\rho_{0}, \rho_{1}\right)} \exp \left[-\rho_{1}(x-1)\right] .
\end{gathered}
$$

In the sector $S_{\varepsilon}$, for $\left|\rho_{0}\right|,\left|\rho_{1}\right| \rightarrow \infty$ we have $\left\|\exp \rho_{0} x\right\|_{L_{q}(0, b)} \leq c(\varepsilon)\left|\rho_{0}\right|^{-\frac{1}{q}}$, 
$\left\|\exp \left[-\rho_{0}(x-b)\right]\right\|_{L_{q}(0, b)} \leq c(\varepsilon)\left|\rho_{0}\right|^{-\frac{1}{q}}, \quad\left\|\exp \rho_{1}(x-b)\right\|_{L_{q}(b, 1)} \leq c(\varepsilon)\left|\rho_{1}\right|^{-\frac{1}{q}}$ and $\left\|\exp \left[-\rho_{1}(x-1)\right]\right\|_{L_{q}(b, 1)} \leq c(\varepsilon)\left|\rho_{1}\right|^{-\frac{1}{q}}$. Hence, for $k \geq 0$ and $\lambda \in S_{\varepsilon}$, we get

$$
\left\|y_{2}^{(k)}\right\|_{L_{1}(0, b) \times L_{q}(b, 1)} \leq c(\varepsilon) \sum_{\nu=0}^{4}|\lambda|^{\frac{1}{2}\left(k-\frac{1}{q}-k_{\nu}\right)}\left(\left|L_{\nu 0} y_{1}\right|+\left|f_{\nu}\right|\right)
$$

To evaluate $\left|L_{\nu 0} y_{1}\right|$, we apply Lemma 1 for $\chi=j+\frac{1}{q}$ and $h=|\lambda|^{-1}$, since

$$
\begin{aligned}
& \left\|u_{1}\right\|_{c} k_{\nu[0, b)} \\
& \quad \leq c \sum_{j=0}^{k}\left(\left|\rho_{0}\right|^{-2\left[1-\frac{1}{2}\left(j+\frac{1}{q}\right)\right]}\left\|u_{1}^{\prime \prime}\right\|_{L_{q}(0, b)}+\left|\rho_{0}\right|^{2\left[\frac{1}{2}\left(j+\frac{1}{q}\right)\right]}\left\|u_{1}\right\|_{L_{q}(0, b)}\right) .
\end{aligned}
$$

This, from (13), gives

$$
\begin{aligned}
& \left\|u_{1}\right\|_{c} k_{\nu[0, b)} \\
& \leq c(\varepsilon) \sum_{j=0}^{k_{\nu}}\left|\rho_{0}\right|^{j+\frac{1}{q}-l}\left(\left\|f_{1}\right\|_{W_{q(0, b)}^{l-2}}+\left|\rho_{0}\right|^{l-2}\left\|f_{1}\right\|_{L_{q}(0, b)}\right)
\end{aligned}
$$

By an analogous argument for $u_{2}$, we obtain

$$
\begin{aligned}
& \left\|u_{2}\right\|_{c}^{k}{ }_{c(b, 1]} \\
& \quad \leq c(\varepsilon) \sum_{j=0}^{k}\left|\rho_{1}\right|^{j+\frac{1}{q}-l}\left(\left\|f_{2}\right\|_{W_{q(0, b)}^{l-2} \times W_{q}^{l-2}(b, 1)}+\left|\rho_{1}\right|^{l-2}\left\|f_{2}\right\|_{L_{q}(b, 1)}\right) .
\end{aligned}
$$

$\operatorname{As}\left|L_{\nu 0} y\right| \leq c\left\|y_{1}\right\|_{c} k_{\nu[0, b) \times c}{ }^{k}{ }_{\nu(b, 1]}$, so

$$
\begin{gathered}
\left|L_{\nu 0} y_{1}\right| \leq c(\varepsilon) \sum_{j=0}^{k}|\lambda|^{\frac{1}{2}\left(-l+j+\frac{1}{q}\right)}\left(\|f\|_{W_{q}^{l-2}(0, b) \times w_{q}^{l-2}(b, 1)}\right. \\
\left.+|\lambda|^{\frac{1}{2}(l-2)}\|f\|_{L_{q}(0, b) \times L_{q}(b, 1)}\right) \\
\leq c(\varepsilon)|\lambda|^{\frac{1}{2}\left(-l+k_{\nu}+\frac{1}{q}\right)}\left(\|f\|_{W_{q}^{l-2}(b, 1)}+|\lambda|^{\frac{1}{2}(l-2)}\|f\|_{L_{q}(0, b) \times L_{q}(b, 1)}\right) \\
\nu=\overline{1,4}
\end{gathered}
$$

Applying (16), we get that inequality (15) gives estimate (7). The uniqueness of the solution of problem (4)-(6) follows from estimate (7). 


\subsection{Coerciveness of the General Problem}

First, consider the following definition.

Definition 1: Boundary value and transmission conditions (2)-(3) are said to be regular if the following hold.

1. $\quad \frac{(-1)^{K}{ }^{\alpha_{11}} \beta_{21}}{\left(\sqrt{a_{0}}\right)^{K}{ }_{1}\left(\sqrt{a_{1}}\right)^{K_{2}}}-\frac{(-1)^{K_{1}} \alpha_{21} \beta_{11}}{\left(\sqrt{a_{0}}\right)^{K}{ }\left(\sqrt{a_{1}}\right)^{K_{1}}} \neq 0$ and $\frac{\alpha_{31} \beta_{41}}{\sqrt{a_{0}}}-\frac{\beta_{31} \alpha_{41}}{\sqrt{a_{1}}} \neq 0$.

2. For some $q \in(1, \infty)$, each functional $T_{\nu}$ is continuous in $W_{q}^{k} \nu(0, b) \times$ $W_{q}^{k} \nu(b, 1)$, where $\nu=\overline{1,4}, 0 \leq k_{\nu} \leq 1$ for $\nu=\overline{1,2}$ and $k_{3}=0$ and $k_{4}=1$.

Remark 2: The above definition coincides with the Yakubov definition ([14], p. 86) in the case where $\alpha_{31}=\alpha_{41}=\beta_{31}=\beta_{41}=0$.

Theorem 2: Suppose the following hold.

1. Boundary and transmission conditions (2)-(3) are regular.

2. Operator $B$, from $W_{q}^{l}(0, b) \times W_{q}^{l}(b, 1)$ into $W_{q}^{l-2}(0, b) \times W_{q}^{l-2}(b, 1)$ is compact, where $l \geq 2$ and $q \in(1, \infty)$.

Then, for all $\lambda \in S_{\varepsilon}$, the operator

$$
\mathcal{L}(\lambda): u \mapsto\left(L_{0} u, L_{10} u, L_{20} u, L_{30} u, L_{40} u\right),
$$

from $W_{q}^{l}(0, b) \times W_{q}^{l}(b, 1)$ into $W_{q}^{l-2}(0, b) \times W_{q}^{l-2}(b, 1) \times \mathbb{C}^{4}$, is a Fredholm operator where

Proof: The operator $\mathcal{L}(\lambda)$ can be represented in the form $\mathcal{L}(\lambda)=\mathcal{L}_{0}(\lambda)+\mathscr{L}_{1}(\lambda)$,

$$
\ell_{0}(\lambda)=\left(-a(x) u^{\prime \prime}(x)-\lambda u(x), u(0), u^{\prime}(1), u(b-0), u^{\prime}(b+0)\right)
$$

and

$$
\ell_{1}(\lambda)=\left(B u, L_{10} u-u(0), L_{20} u-u^{\prime}(1), L_{30} u-u(b-0), L_{40} u-u^{\prime}(b+0)\right) .
$$

From Theorem 1, it follows that $\ell_{0}(\lambda)$ is an isomorphism for $\lambda \in S_{\varepsilon}$. Hence, it is a Fredholm operator. By virtue of hypotheses 1 and $2, \ell_{1}(\lambda)$, from $W_{q}^{l}(0, b) \times W_{q}^{l}(b, 1)$ into $W_{q}^{l-2}(0, b) \times W_{q}^{l-2}(b, 1) \times \mathbb{C}^{4}$, is compact. Then, by a theorem on perturbation of Fredholm operators (see [7]), $\mathcal{L}(\lambda)=\ell_{0}(\lambda)+\mathcal{L}_{1}(\lambda)$ is a Fredholm operator.

Theorem 3: Suppose that conditions 1 and 2 of Theorem 2 are satisfied. Then, for any $\varepsilon>0$, there is $R_{\varepsilon}>0$ such that for any complex number $\lambda$ where $\lambda \in S_{\varepsilon}$ and $|\lambda|>R_{\varepsilon}$, the operator

$$
\mathcal{L}(\lambda): u \rightarrow\left(L(\lambda) u, L_{1}(\lambda) u, L_{2}(\lambda) u, L_{3}(\lambda) u, L_{4}(\lambda) u\right)
$$

from $W_{q}^{l}(0, b) \times W_{q}^{l}(b, 1)$ into $W_{q}^{l-2}(0, b) \times W_{q}^{l-2}(b, 1) \times \mathbb{C}^{4}, \quad$ is an isomorphism. Furthermore, for such a $\lambda$, estimate (7) holds for the solution of problem (1)-(3).

Proof: By displacing the perturbed terms of problem (1)-(3) to the right-hand side members, and by applying Theorem 1 to the obtained problem, we find that

$$
\sum_{k=0}^{l}|\lambda|^{\frac{1}{2}(l-k)}\|u\|_{W_{q}^{k}(0, b) \times W_{q}^{k}(b, 1)}
$$




$$
\begin{aligned}
& \leq C(\varepsilon)\left(\|f\|_{W_{q}^{l-2}(0, b) \times W_{q}^{l-2}(b, 1)}+|\lambda|^{\frac{1}{2}(l-2)}\|f\|_{L_{q}(0, b) \times L_{q}(b, 1)}\right. \\
& \quad+\sum_{\nu=1}^{4}|\lambda|^{\frac{1}{2}\left(l-k_{\nu}-\frac{1}{q}\right)}\left|f_{\nu}\right|+\|B u\|_{W_{q}^{l-2}(0, b) \times W_{q}^{l-2}(b, 1)} \\
& \left.+|\lambda|^{\frac{1}{2}(l-2)}\|B u\|_{L_{q}(0, b) \times L_{q}(b, 1)}+\sum_{\nu=1}^{4}|\lambda|^{\frac{1}{2}\left(l-k_{\nu}-\frac{1}{q}\right)}\left|T_{\nu} u\right|\right) .
\end{aligned}
$$

The above inequality, Lemma 2.7 from [14] and the continuity of $T_{\nu}$ give

$$
\begin{aligned}
& \sum_{k=0}^{l}|\lambda|^{\frac{1}{2}(l-k)}\|u\|_{W_{q}^{k}(0, b) \times W_{q}^{k}(b, 1)} \\
& \quad \leq C(\varepsilon)\left(\|f\|_{W_{q}^{l-2}(0, b) \times W_{q}^{l-2}(b, 1)}+|\lambda|^{\frac{1}{2}(l-2)}\|f\|_{L_{q}(0, b) \times L_{q}(b, 1)}\right. \\
& +\sum_{\nu=1}^{4}|\lambda|^{\frac{1}{2}\left(l-k_{\nu}-\frac{1}{q}\right)}\left|f_{\nu}\right| \\
& \quad+c(\varepsilon)\left(\delta+c(\delta)|\lambda|^{\left.-\frac{1}{2 q}\right)} \sum_{k=0}^{l}|\lambda|^{\frac{1}{2}(l-k)}\|u\|_{W_{q}^{k}(0, b) \times W_{q}^{k}(b, 1)}\right) .
\end{aligned}
$$

Choosing $\delta$ such that $c(\varepsilon)\left(\delta+c(\delta)|\lambda|^{-\frac{1}{2 q}}\right)<1$, we get inequality (7), which implies that $\mathcal{L}(\lambda)$ is injective. Since operator $B$, from $W_{q}^{l}(0, b) \times W_{q}^{l}(b, 1)$ into $W_{q}^{l-2}(0, b) \times$ $W_{q}^{l-2}(b, 1)$, is compact, and since according to Theorem $2 \quad \mathcal{L}(\lambda): W_{q}^{l}(0, b) \times$ $W_{q}^{q}(b, 1) \rightarrow W_{q}^{l-2}(0, b) \times W_{q}^{l-2}(b, 1) \times \mathbb{C}^{4}$ is a Fredholm operator, then by a Fredholm alternative $\mathcal{L}(\lambda)$ is surjective. Therefore, it is an isomorphism.

\section{Completeness of Root Functions}

In the space $L_{2}(0, b) \times L_{2}(b, 1)$, consider the operator $\mathcal{L}$ defined as follows.

$$
\begin{gathered}
\mathcal{L} u=-a(x) u^{\prime \prime}(x)+(B u)(x) \\
D(\mathcal{L})=\left(W_{2}^{2}(0, b) \times W_{2}^{2}(b, 1), L_{\nu} u=0, \nu=\overline{1,4}\right) .
\end{gathered}
$$

The root functions of operator $\mathcal{L}$ are root functions of the following problem:

$$
\left\{\begin{aligned}
L(\lambda) u & =0 \\
L_{\nu}(\lambda) u & =0, \nu=\overline{1,4}
\end{aligned}\right.
$$

To establish the completeness of the root functions of $\mathcal{L}$, we shall use a theorem given in [14] (Theorem 3.6, with $n=1$ ). This theorem is actually a variation of the well- 
known theorem of N. Danford ad J.T. Schwartz [3]. Consider the following.

Theorem 4: Suppose that the conditions given below are satisfied.

1. There exist two Hilbert spaces, $H$ and $H_{1}$, with the compact embedding $H \subset H_{1}$, and $\left.\bar{H}_{1}\right|_{H}=H$.

2. The embedding operator $g$ belongs to $\sigma_{p}\left(H_{1}, H\right)$ for some $p>0$.

3. The linear operator $A$ from $H_{1}$ and $H$ is bounded.

4. There exists a set of rays $l_{k}$, in the complex plane such that angles between the neighboring rays are less than $\frac{\pi}{p}$; and there exists a number $m \in \mathbb{N}$ such that $\|R(\lambda, A)\|_{B\left(H, H_{1}\right)} \leq c|\lambda|^{m}$, with $\lambda \in l_{k}$ and with $|\lambda| \rightarrow \infty$.

Then the spectrum of operator $A$ is discrete and the system of root vectors of operator $A$ is complete in the space $H_{1}$.

Applying the method used in proving Theorem 2.1 in [14] and Theorem 3, we can prove the following lemma.

Lemma 3:

$$
\left.\overline{\left(W_{2}^{2}(0, b) \times W_{2}^{2}(b, 1), L_{\nu} u=0, \nu=\overline{1,4}\right)}\right|_{L_{2}(0, b) \times L_{2}(b, 1)}=L_{2}(0, b) \times L_{2}(b, 1) .
$$

Theorem 5: Suppose that the conditions below hold.

1. Boundary and transmission conditions of problem (17) are regular.

2. Operator $B$, from $W_{2}^{2}(0, b) \times W_{2}^{2}(b, 1)$ into $L_{2}(0, b) \times L_{2}(b, 1)$ is compact.

Then the spectrum of problem (17) is discrete, and the system of root functions of problem (17) is complete in $\left(W_{2}^{2}(0, b) \times W_{2}^{2}(b, 1), L_{\nu} u=0, \nu=\overline{1,4}\right)$ and, therefore, in $L_{2}(0, b) \times L_{2}(b, 1)$.

Proof: Set $H=L_{2}(0, b) \times L_{2}(b, 1) \quad$ and $\quad H_{1}=\left(W_{2}^{2}(0, b) \times W_{2}^{2}(b, 1), \quad L_{\nu} u=0\right.$, $\nu=\overline{1,4})$. Since embeddings $W_{2}^{2}(0, b) \subset L_{2}(0, b)$ and $W_{2}^{2}(b, 1) \subset L_{2}(b, 1)$ are compact [12], then embeddings $W_{2}^{2}(0, b) \times W_{2}^{2}(b, 1) \subset L_{2}(0, b) \times L_{2}(b, 1)$ is compact. Using hypothesis 1 and Lemma 3 , we find $\left.\bar{H}_{1}\right|_{H}=H$. take $p=\frac{1}{2}+\delta$, where $\delta$ is an arbitrary positive number. From [12], we get $S_{j}\left(g, W_{2}^{2}(0, b), L_{2}(0, b)\right) \sim j^{-2}$ and $S_{j}\left(g, W_{2}^{2}(b, 1), L_{2}(b, 1)\right) \sim j^{-2}$, and so, $g \in \sigma_{\frac{1}{2}+\delta}\left(W_{2}^{2}(0, b), L_{2}(0, b)\right)$ and $g \in \sigma_{\frac{1}{2}+\delta}$

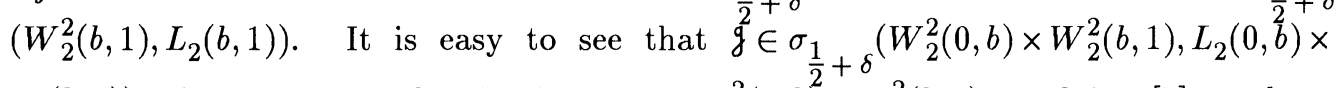
$\left.L_{2}(b, 1)\right)$. Since $H_{1}$ is a closed subspace in $W_{2}^{2}(0, b) \times W_{2}^{2}(b, 1)$, applying [5], we have $g \in \sigma_{\frac{1}{2}+\delta}\left(H_{1}, H\right)$. It is obvious that operator $\mathcal{L}$ is bounded from $H_{1}$ into $H$. From Theorem 3, we see that in the sector $S_{\varepsilon}$ we have $\|R(\lambda, \mathcal{L})\|_{B\left(H, H_{1}\right)} \leq c(\varepsilon)$, for $|\lambda| \rightarrow \infty$. From this sector $S_{\varepsilon}$, take two rays $l_{1}$ and $l_{2}$ centered at the origin and choose a number $\delta>0$ such that the angle between the two rays is less than $\frac{\pi}{\frac{1}{2}+\delta}$.
Since all conditions of Theorem 4 are satisfied we get the desired result.

\section{A Nonlocal Parabolic Transmission Problem}

\subsection{Correct Solvability}

In $[0, T] \times([0,1] \backslash b)$, consider the equation 


$$
\frac{\partial u(t, x)}{\partial t}+a(x) \frac{\partial^{2} u(t, x)}{\partial x^{2}}+(B u(t, \cdot))(x)=0
$$

with the functional boundary conditions:

$$
\left\{\begin{array}{c}
L_{10} u=\alpha_{11} u^{\left(K_{1}\right)}(t, 0)+\beta_{11} u^{\left(K_{1}\right)}(t, 1)+\sum_{p=1}^{N} \delta_{1 p} u^{\left(K_{1}\right)}\left(t, x_{1 p}\right)+T_{1} u(t, \cdot)=0 \\
L_{20} u=\alpha_{21} u^{\left(K_{2}\right)}(t, 0)+\beta_{21} u^{\left(K_{2}\right)}(t, 1)+\sum_{p=1}^{M} \delta_{2 p} u^{\left(K_{2}\right)}\left(t, x_{2 p}\right)+T_{2} u(t, \cdot)=0
\end{array}\right.
$$

with the functional transmission conditions:

$$
\left\{\begin{array}{r}
L_{30} u=\alpha_{31} u(t, b-0)+\beta_{31} u(t, b+0)+T_{3} u(t, \cdot)=0 \\
L_{40} u=\alpha_{41} u^{\prime}(t, b-0)+\beta_{41} u^{\prime}(t, b+0)+T_{4} u(t, \cdot)=0
\end{array}\right.
$$

and with the initial condition:

$$
u(0, x)=\varphi_{0}(x)
$$

where $a(x)=\left\{\begin{array}{c}a_{0} \text { for } x \in[0, b) \\ a_{1} \text { for } x \in(b, 1] .\end{array} \quad x_{1 p}, x_{2 p} \in(0, b) ; B\right.$ is a linear operator; each $T_{\nu}$ is a linear functional $(\nu=\overline{1,4}) ; \alpha_{j 1}, \beta_{j 1}, \delta_{i p} \in \mathbb{C} ; K_{i}=0,1 ; i=\overline{1,2} ; j=\overline{1,4} ; p=\overline{1, N}$.

Theorem 6: Let the following conditions be satisfied.

1. $\quad a_{i} \neq 0$ and $\left|\arg a_{i}\right|>\frac{\pi}{2}$.

2. $\quad \frac{(-1)^{K}{ }^{\alpha_{11} \beta_{21}}}{\left(\sqrt{a_{0}}\right)^{K}{ }_{1}\left(\sqrt{a_{1}}\right)^{K_{2}}}-\frac{(-1)^{K} \alpha_{21} \beta_{11}}{\left(\sqrt{a_{0}}\right)^{K}{ }^{2}\left(\sqrt{a_{1}}\right)^{K_{1}}} \neq 0$ and $\frac{\alpha_{31} \beta_{41}}{\sqrt{a_{0}}}-\frac{\beta_{31} \alpha_{41}}{\sqrt{a_{1}}} \neq 0$.

3. The operator $B$, from $W_{2}^{2}(0, b) \times W_{2}^{2}(b, 1)$ into $L_{2}(0, b) \times L_{2}(b, 1)$ is compact.

4. Each functional $T_{\nu}, \nu=\overline{1,4}$, is continuous in $W_{2}^{k} \nu(0, b) \times W_{2}^{k \nu}(b, 1)$.

5. $\varphi_{0} \in\left(W_{2}^{2}(0, b) \times W_{2}^{2}(b, 1), L_{\nu} u=0, \nu=\overline{1,4}\right)$.

Then problem (18)-(21) has a unique solution $u$ in

$$
C\left([0, T], L_{2}(0, b) \times L_{2}(b, 1)\right) \cap C^{1}\left([0, T], W_{2}^{2}(0, b) \times W_{2}^{2}(b, 1), L_{2}(0, b) \times L_{2}(b, 1)\right) ;
$$

and we have the following inequalities:

$$
\|u(t)\|_{L_{2}(0, b) \times L_{2}(b, 1)} \leq c\left\|\varphi_{0}\right\|_{W_{2}^{2}(0, b) \times W_{2}^{2}(b, 1)}, t \in(0, T]
$$

and

$$
\begin{gathered}
\left\|u^{\prime}(t)\right\|_{L_{2}(0, b) \times L_{2}(b, 1)}+\|u(t)\|_{W_{2}^{2}(0, b) \times W_{2}^{2}(b, 1)} \\
\leq c \cdot t^{-1}\left\|\varphi_{0}\right\|_{W_{2}^{2}(0, b) \times W_{2}^{2}(b, 1)}, \quad t \in(0, T] .
\end{gathered}
$$


Proof: Let $A$ denote the operator defined on $L_{2}(0, b) \times L_{2}(b, 1)$ by $A u(x)=$ $-a(x) u^{\prime \prime}(x)$ with $D(A)=\left(W_{2}^{2}(0, b) \times W_{2}^{2}(b, 1), L_{\nu} u=0, \nu=\overline{1,4}\right)$. Then problem (18)$(21)$, in $L_{3}(0, b) \times L_{2}(b, 1)$, can be rewritten as follows.

$$
\left\{\begin{array}{c}
u^{\prime}(t)=A u(t)-B u(t) \\
u(0)=\varphi_{0}
\end{array}\right.
$$

where $u(t)=u(t, \cdot)$ and $f(t)=f(t, \cdot)$ are functions with values in $L_{2}(0, b) \times L_{2}(b, 1)$, $\varphi_{0}=\varphi_{0}(\cdot) \in L_{2}(0, b) \times L_{2}(b, 1)$. Using Theorem 3 in sector $S_{\varepsilon}$, we get $\|R(\lambda, A)\| \leq$ $c|\lambda|^{-1},|\lambda| \rightarrow \infty$. From hypothesis 1 , the number $\varepsilon>0$ can be chosen sufficiently small so that for some $\alpha>0$ we have

$$
\|R(\lambda, A)\| \leq c|\lambda|^{-1},|\arg \lambda| \leq \frac{\pi}{2}+\alpha,|\lambda| \rightarrow \infty
$$

We know that operator $B$ is compact, from $W_{2}^{2}(0, b) \times W_{2}^{2}(b, 1)$, into $L_{2}(0, b) \times$ $L_{2}(b, 1)$, and operator $R(\lambda, A)$ is bounded, from $L_{2}(0, b) \times L_{2}(b, 1)$ into $W_{2}^{2}(0, b) \times$ $W_{2}^{2}(b, 1)$ (by Theorem 3 ). Consequently, operator $T=B R(\lambda, A)$ is compact, from $L_{2}(0, b) \times L_{2}(b, 1)$ into $L_{2}(0, b) \times L_{2}(b, 1)$. Now, by Lemma $3, D(A)$ is dense in $L_{2}(0, b) \times L_{2}(b, 1)$; and since the space $L_{2}(0, b) \times L_{2}(b, 1)$ has a basis and is reflexive, then, by Lemma 2.7 from [14] we have that for arbitrary $\varepsilon>0$ and for arbitrary $u \in D(A)$

$$
\begin{aligned}
& \|B u\|_{L_{2}(0, b) \times L_{2}(b, 1)} \\
& \quad \leq \varepsilon\|(A-\lambda I) u\|_{L_{2}(0, b) \times L_{2}(b, 1)}+c(\varepsilon)\|u\|_{L_{2}(0, b) \times L_{2}(b, 1)} .
\end{aligned}
$$

Since $\varphi_{0} \in D(A)$, then Lemma 2.7 from [14] can be applied to problem (22)-(23), which gives the desired result.

\subsection{Completeness of Elementary Solutions}

It is not difficult to show (see Lemma 0.1 from [14]) that a function $U_{j}$, given by the formula

$$
U_{j}(t)=e^{\lambda_{0} t}\left(\frac{t^{k}}{k !} u_{0}+\frac{t^{k-1}}{(k-1) !} u_{1}+\ldots+\frac{t}{1 !} u_{k-1}+u_{k}\right),
$$

where $j=\overline{0, k}$, becomes a solution to equation (22) if and only if $u_{0}, u_{1}, \cdots, u_{k}$ is a chain of root functions of the operator $A+B$ corresponding to eigenvalue $\lambda_{0}$. A solution of form (24) is called an elementary solution to equation (22).

Theorem 7: Suppose that all conditions of Theorem 6 are satisfied. Then problem (18)-(21) has a unique solution:

$$
u \in C\left([0, T], L_{2}(0, b) \times L_{2}(b, 1)\right) \cap C^{1}\left((0, T], W_{2}^{2}(0, b) \times W_{2}^{2}(b, 1), L_{2}(0, b) \times L_{2}(b, 1)\right) ;
$$

and there exists a set of numbers, $c_{j n}$, such that

$$
\lim _{n \rightarrow \infty} \sup _{t \in[0, T]}\left\|u(t, \cdot)-\sum_{j=1}^{n} c_{j n} u_{j}(t, \cdot)\right\| L_{2}(0, b) \times L_{2}(b, 1)=0
$$


and

$$
\begin{gathered}
\lim _{n \rightarrow \infty} \sup _{t \in(0, T]} t\left(\left\|u_{t}^{\prime}(t, \cdot)-\sum_{j=1}^{n} c_{j n} u_{j t}^{\prime}(t, \cdot)\right\|_{L_{2}(0, b) \times L_{2}(b, 1)}\right. \\
\left.+\left\|u(t, \cdot)-\sum_{j=1}^{n} c_{j n} u_{j}(t, \cdot)\right\|_{W_{2}^{2}(0, b) \times W_{2}^{2}(b, 1)}\right)=0,
\end{gathered}
$$

where $u$ is a solution of problem (18)-(21) and each $u_{j}$ is an elementary solution of problem (18)-(20).

Proof: By Theorem 6, we get the existence and uniqueness of the solution to problem (18)-(21); and by Theorem 5, the completeness of root functions of problem (17) is guaranteed. Therefore, if we denote by $\lambda_{j}, j=\overline{1, \infty}$, the eigenvalues of problem (17), taking into consideration their order of algebraic multiplicity, there exists a set of numbers $c_{j n}$, such that

$$
\lim _{n \rightarrow \infty}\left\|\varphi_{0}-\sum_{j=1}^{n} c_{j n} u_{j k}\right\|_{W_{2}^{2}(0, b) \times W_{2}^{2}(b, 1)}=0
$$

where $u_{j 0}, u_{j 1}, \cdots, u_{j k}$ form some chain of root functions of problem (17) corresponding to the eigenvalues $\lambda_{j}$. On the other hand, using Theorem 6 , we find that problem (18)-(20), with the initial condition $u(0, x)=\varphi_{0}(x)-\sum_{j=1}^{n} c_{j n} u_{j k_{j}}(x)$, has a unique
solution

$$
(t, x) \rightarrow v(t, x)=u(t, x)-\sum_{j=1}^{n} c_{j n} u_{j}(t, x)
$$

in the space

$$
C\left([0, T], L_{2}(0, b) \times L_{2}(b, 1)\right) \cap C^{1}\left((0, T], W_{2}^{2}(0, b) \times W_{2}^{2}(b, 1), L_{2}(0, b) \times L_{2}(b, 1)\right) .
$$

We also have the following inequalities:

$$
\left\|u(t, \cdot)-\sum_{j=1}^{n} c_{j n} u_{j}(t, \cdot)\right\|_{L_{2}(0, b) \times L_{2}(b, 1)} \leq c\left\|\varphi_{0}-\sum_{j=1}^{n} c_{j n} u_{j k}\right\|_{W_{2}^{2}(0, b) \times W_{2}^{2}(b, 1)}
$$

and

$$
\begin{aligned}
& \left\|u_{t}^{\prime}(t, \cdot)-\sum_{j=1}^{n} c_{j n} u_{j t}^{\prime}(t, \cdot)\right\|_{L_{2}(0, b) \times L_{2}(b, 1)} \\
& +\left\|u(t, \cdot)-\sum_{j=1}^{n} c_{j n} u_{j}(t, \cdot)\right\|_{W_{2}^{2}(0, b) \times W_{2}^{2}(b, 1)}
\end{aligned}
$$




$$
\leq c \cdot t^{-1}\left\|\varphi_{0}-\sum_{j=1}^{n} c_{j n} u_{j k}\right\|_{W_{2}^{2}(0, b) \times W_{2}^{2}(b, 1)} .
$$

Therefore, the proof of the theorem is complete.

\section{References}

[1] Agranovich, M.S. and Vishik, M.I., Elliptic problems with a parameter and parabolic problems of general type, Russian Math. Survey 19 (1964), 53-159.

[2] Besov, O.V., Il'in, V.P. and Nikolskii, S.M., Integral Representations and Embedding Theorems, Halsted Press, New York I 1978.

[3] Danford, N. and Schwartz, J.T., Linear Operators. Part II: Spectral Theory, Interscience 1963.

[4] Dezin, A.A., General Questions of the Boundary Value Problem Theory, Nauka, Moscow 1980 (in Russian).

[5] Gohberg, I.C. and Krein, M.G., Introduction to the Theory of Linear Non-Selfadjoint Operators, Amer. Math. Soc., Providence, RI 1969.

[6] Goureev, G.M. and Kovalenko, A.I., Completeness criterion for root subspaces of derivation operator with abstract boundary conditions, Mat. Zametki 4:30 (1981), 543-552 (in Russian).

[7] Kato, T., Perturbation Theory for Linear Operators, Springer-Verlag 1966.

[8] Krein, S.G., Linear Differential Equations in Banach Space, Providence 1971.

[9] Naimark, M.A., Linear Differential Operators, Ungar, New York 1967.

[10] Shkalikov, A.A., Boundary value problems for ordinary differential equations with a parameter in boundary conditions, J. Soviet. Math. 33 (1986), 1311-1342.

[11] Shkalikov, A.A., Basis properties of root vectors for ordinary differential operators with integral boundary conditions, Vestnik Mosk. Univ. Serie: Math-Mech. Series 6 (1982), 12-21 (in Russian).

[12] Triebel, H., Interpolation Theory. Function Spaces. Differential Operators, North-Holland, Amsterdam 1978.

[13] Voitovich, N.N., Katsenelenbaum, B.Z. and Sivov, A.N., Generalized Method of Eigen-Oscillations in the Diffraction Theory, Nauka, Moscow 1977 (in Russian).

[14] Yakubov, S.Ya., Completeness of Root Functions of Regular Differential Operators, Longman 1994. 


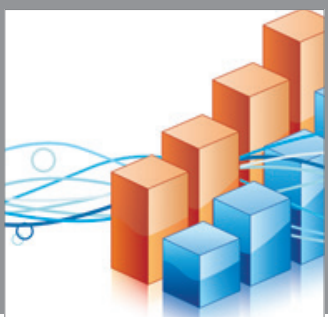

Advances in

Operations Research

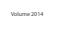

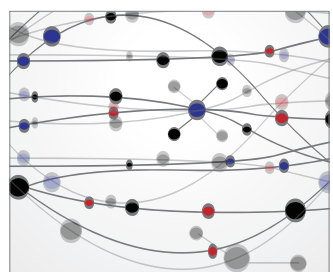

\section{The Scientific} World Journal
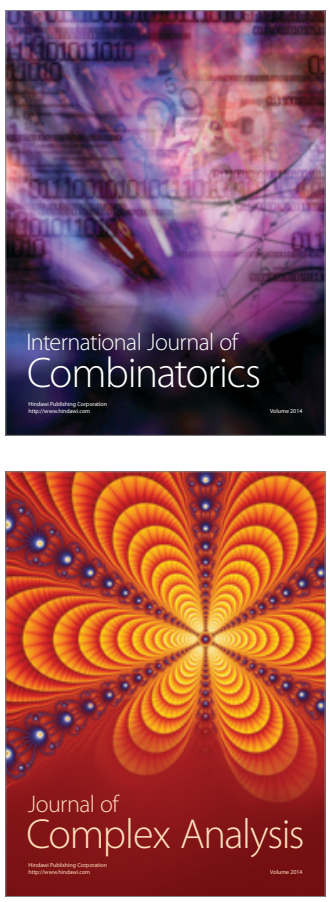

International Journal of

Mathematics and

Mathematical

Sciences
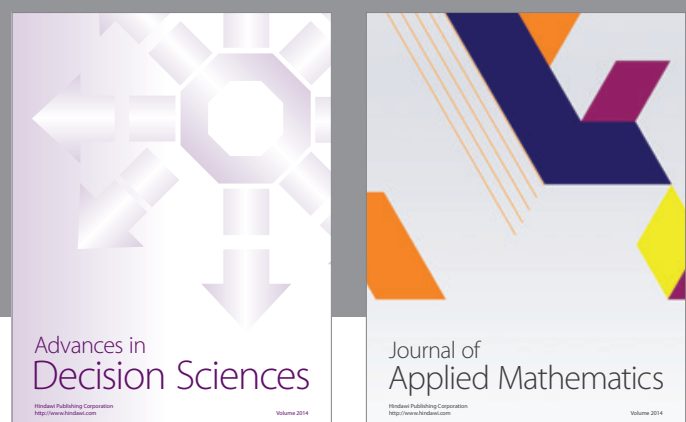

Journal of

Applied Mathematics
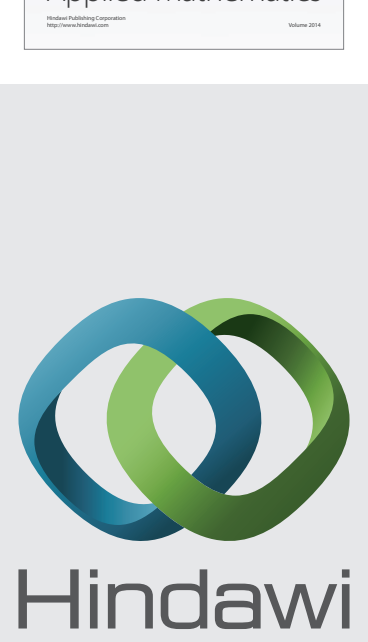

Submit your manuscripts at http://www.hindawi.com
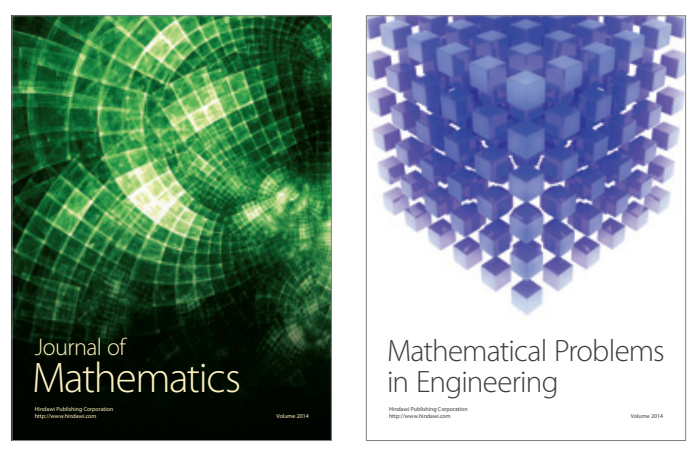

Mathematical Problems in Engineering
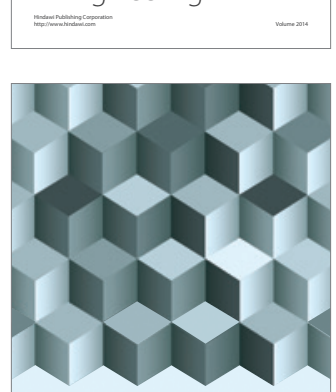

Journal of

Function Spaces
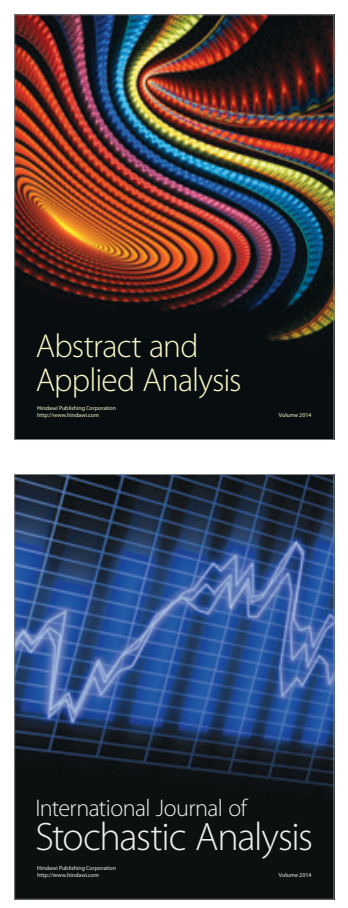

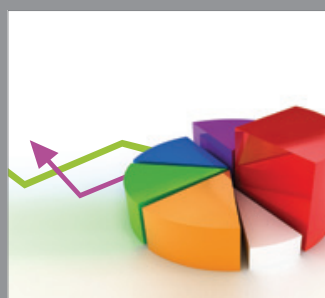

ournal of

Probability and Statistics

Promensencen
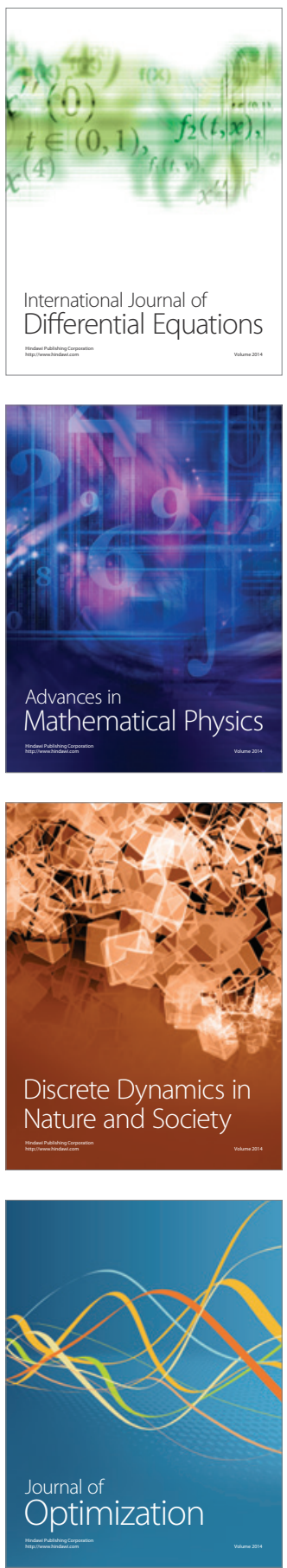